\section{Metabolite-mediated misfolding}

The three-dimensional conformation of a protein is specified by its amino-acid sequence, so mutations can lead to aberrant folding. But why do normal, mutation-free proteins become misfolded? Resolving this conundrum would improve our understanding of the sporadic form of Alzheimer's disease, in which wild-type amyloid $\beta$-peptides $(A \beta)$ misfold and aggregate to form pathogenic plaques in the brain.

One possibility is that modification by abnormal metabolites causes wild-type $A \beta$ to misfold. Recently established links between Alzheimer's disease, high cholesterol levels and inflammation prompted a team of investigators led by Jeffery Kelly to focus on the $A \beta$-modifying potential of metabolites that form through the reaction of cholesterol with ozone, which is generated during inflammation.

A key property of these cholesterol metabolites is that they possess an aldehyde group, which could covalently modify amines in $A \beta$. This attachment would markedly increase the hydrophobicity of $A \beta$, potentially increasing the likelihood of misfolding. To assess this possibility, several cholesterol metabolites were individually incubated with $A \beta$. Two such metabolites dubbed compounds 1 and 2 - caused a concentration-dependent increase in the rate of amyloidogenesis and were shown to be present in human brains.

Once formation of aggregates in the in vitro assay had ceased, the authors measured the amount of soluble $A \beta$ that remained in the reaction mixture. This provided an estimate of the 'critical concentration' for aggregation in the presence of compound 1 or 2 . The derived maximal value of $90 \mathrm{nM}$ is much lower than that for metabolite-free $A \beta$, which might explain how physiological concentrations of $A \beta$ (typically in the nanomolar range) could form amyloid plaques in individuals that lack predisposing

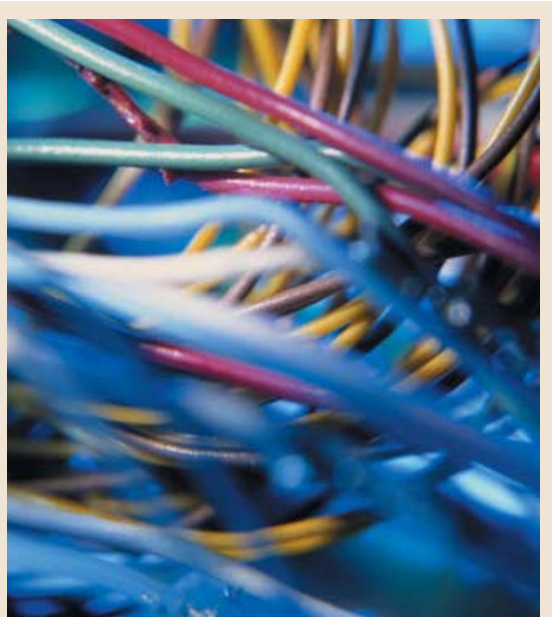

mutations. The authors suggest that atherosclerosis-related inflammation causes ozonolysis of cholesterol, transiently increasing the concentrations of highly reactive metabolites that in turn initiate amyloidogenesis.

Suzanne Farley

(0) References and links

ORIGINAL RESEARCH PAPER Zhang, Q. et al. Metaboliteinitiated protein misfolding may trigger Alzheimer's disease. Proc. Natt Acad. Sci. USA 101, 4752-4757 (2004)

\title{
Special agents provide early warnings
}

The early diagnosis of Alzheimer's disease the most common cause of dementia in the elderly - remains problematic. Now, Nobuyuki Okamura and colleagues report a potential way to detect early changes in patients who are at risk of developing Alzheimer's disease through the non-invasive imaging of senile plaques - a pathological change that precedes the onset of cognitive deterioration.

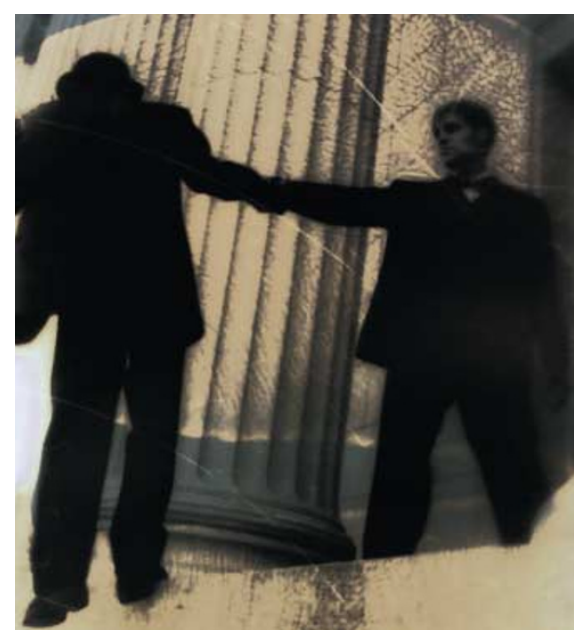

Senile plaques contain amyloid- $\beta$ (A $\beta)$ peptide, levels of which are elevated in early dementia. The initial stages of Alzheimer's disease are characterized by extensive deposition of diffuse plaques and the formation of neurofibrillary tangles in the entorhinal cortex. An agent that can selectively recognize amyloid fibrils and that can permeate the blood-brain barrier might allow clinicians to visualize these initial pathological changes in vivo using positron emission tomography (PET) or single-photon emission computed tomography (SPECT).

Okamura et al. screened many derivatives of styrylbenzoxazole for those that that have a high binding affinity to amyloid fibrils. One of these derivatives, 6-(2-fluoroethoxy)-2-[2-(4-methylaminophenil) ethenyl]benzoxazole (BF-168), selectively recognized senile plaques and neurofibrillary tangles: in vitro binding assays showed that it had a high binding affinity with synthetic $\mathrm{A} \beta$ aggregates, and neuropathological staining showed evidence for binding of BF-168 to both neuritc and diffuse plaques in brain sections from patients with Alzheimer's disease.

The authors went on to test whether BF-168 could be used for in vivo imaging of amyloid deposits in the brain. In two transgenic mouse models of Alzheimer's disease, fluorescent microscopy and autoradiography showed that the compound bound selectively and specifically to both compact and, more importantly, diffuse $A \beta$ deposits in the neocortex, hippocampus and entorhinal cortex. This, together with the high initial uptake of $\left[{ }^{18} \mathrm{~F}\right] \mathrm{BF}-168$ and fast brain washout, indicates that this agent might be an ideal imaging probe.

Further work is needed to demonstrate the safety and efficacy of BF-168 in humans. However, if it can be successfully used in the clinic, in vivo imaging of amyloid plaques using BF-168 offers hope for the identification of individuals who are at risk of developing Alzheimer's desease, and in turn, for the application of potential preventative treatments for pre-symptomatic patients.

\section{(4) References and links}

Alison Rowan, Copy Editor,

ORIGINAL RESEARCH PAPER Okamura, N. et al.

Styrylbenzoxazole derivatives for in vivo imaging of amyloid plaques in the brain. J. Neurosci. 24, 2535-2541 (2004)

FURTHER READING Sisodia, S. S. \& St George-Hyslop, P. $\gamma$-Secretase, Notch, $A \beta$ and Alzheimer's disease: where do the presenilins fit in? Nature Rev. Neurosci. 3, 281-290 (2002) 